\title{
Performance assessment of embolic protection filters for carotid artery stenting
}

\author{
E. A. Finol ${ }^{1,2}$, C. M. Scotti ${ }^{2}$, I. Verdinelli ${ }^{3}$, C. H. Amon ${ }^{1,2,4}$ \\ \& M. H. Wholey ${ }^{5}$ \\ ${ }^{1}$ Institute for Complex Engineered Systems, Carnegie Mellon University \\ ${ }^{2}$ Department of Biomedical Engineering, Carnegie Mellon University \\ ${ }^{3}$ Department of Statistics, Carnegie Mellon University \\ ${ }^{4}$ Department of Mechanical Engineering. Carnegie Mellon University \\ ${ }^{5}$ Pittsburgh Vascular Institute, University of Pittsburgh Medical Center, \\ U.S.A.
}

\begin{abstract}
Stroke is the third leading cause of death in the United States, accounting for 1.5 deaths reported per 1000 people. Carotid artery stenting (CAS) with cerebral protection is slowly becoming the gold standard for treatment of carotid artery occlusive disease in high risk patients. CAS is based on the selective cannulation of the common carotid artery by means of an introducer sheath or guiding catheter and the deployment of a wire mesh (stent) to treat the occluded artery segment. The goal for CAS is the prevention of stroke and its efficacy depends greatly on the periprocedural complications. The major concern with CAS is its potential to produce emboli that may translate into a severe neurological disorder. In this regard, several cerebral protection devices (CPDs) have been developed recently as an adjunct to CAS, with the primary function of capturing the plaque particles released from the site of vessel injury to prevent neurological events. A category of CPD that has received recent attention due to its ability to allow continued distal perfusion is the embolic protection filter. We tested in vitro one FDA approved (RX Accunet Embolic Protection System, Guidant Corporation, Indianapolis, IN) and two investigational (FilterWire EZ, Boston Scientific, Natick, MA and Angioguard XP, Cordis Corp., Coral Gables, FL) devices of this kind. The objective of this study was to assess the effectiveness of emboli capture of the devices, investigate potential intangible failure modes and complications, and set a baseline of desirable design parameters for future generations of embolic protection filters. None of the devices tested completely prevented embolization into the artery model. Overall, the RX Accunet device had the best filtration performance, failing to capture $0.16 \%$ of plaque particles when deployed in an artery model of $5.5 \mathrm{~mm}$ in diameter. Several complications related to device retrieval were detected in all devices on any given set of testing scenarios. Crossing profile, opening/closing mechanics and pore size were among the key design variables required for improved device designs.
\end{abstract}

Keywords: cerebral protection device, embolic protection, carotid artery stenting. 


\section{Introduction}

While carotid artery stenting (CAS) has been growing at a rate of $20 \%$ per year [1] and has reported encouraging initial and mid-term results, there is healthy skepticism regarding the efficacy and safety of the technique among the vascular community [2-6]. Castriota and others have shown that neurological events are reduced by as much as $79 \%$ with the use of a cerebral protection device $[7,8]$. However, the World Registry reports a $2.23 \%$ rate of strokes and procedurerelated deaths in 4,221 cases of CAS with cerebral protection [9]. Unfortunately, none of the currently available CPDs completely prevents embolization into the carotid artery, and not all patients are able to tolerate the local flow conditions generated by the deployment of the device in the vicinity of the stenosis.

The earliest investigation to report on the effectiveness of a CPD for CAS is an ex vivo analysis performed with the first-generation Neuroshield filter (Mednova, Ireland) in a human carotid plaque specimen under steady flow conditions [10]. The authors showed that the filter captured $88 \%$ of total particles released during CAS, and also provided the first indications of design problems related to pore size, filter recovery, and profile conformity of the filter to the artery wall. Reimers et al [8] performed the first large consecutive series of CAS using the Angioguard XP, Neuroshield, and FilterWire EX filters, reporting a $2.3 \%$ rate of major adverse cardiac event in a 30-day follow-up period. Castriota et al [7] detected vessel spasm of the intracranial carotid artery related to the protection device in $7.3 \%$ of the cases studied. In a more recent study, Bosiers and others [11] report a 92\% procedural success with the FilterWire EX and 5\% delivery failure due to severe internal carotid artery (ICA) angulation in 100 consecutive patients. However, these studies were aimed at evaluating the feasibility of CPD utilization in CAS and are not based on a performance comparison among CPDs in a clinical or experimental setting.

In the absence of an animal model for testing CPDs, Müller-Hülsbeck and associates designed an in vitro bench-top model and compared the performance of several CPDs based on the percentage of emboli missed by the devices [1216]. The authors first tested the Angioguard filter and the GuardWire balloon as supplied by the manufacturers, and also in combination with hydrodynamic aspiration catheters. Their results show that the Angioguard filter alone performed best, failing to capture $1.28 \%$ of emboli. The subsequent studies $[14,15]$ tested a total of five filters: Angioguard, FilterWire EX, Neuroshield, Trap Vascular Filtration System (Microvena, White Bear Lake, MN), and the GuardWire Plus, an improved design of the first-generation device. These devices were tested in the same flow-loop system while using either human plaque material or PVA particles to simulate embolization during CAS. The outcome consistently showed the Neuroshield filter to be the most effective $(0.8$ $-4.1 \%$ of missed particles) and also shed light into the potential design problems of the FilterWire EX that required repositioning of the filter in all experiments to prevent a gap between the device and the artery wall. An improvement in the flow-loop system was made by modeling the carotid artery with anatomical variants that include an elongated ICA and a kinked ICA, both of $5.0 \mathrm{~mm}$ in 
internal diameter [12]. None of the devices completely prevented embolization and there was an increase in non-captured emboli with the introduced anatomical variants in comparison with normal anatomy models. A recent study [16] compared an in-house CPD designed by the authors with five other devices, demonstrating that the new porous-membrane-covered stent was the most effective in capturing PVA particles $(2.9 \%$ missed $)$ and human plaque material $(0 \%$ missed $)$ into the ICA.

The objective of the present work is to assess the effectiveness of three commonly utilized embolic protection filters by testing them in vitro under simulated peak systolic flow conditions. The current work looks to advance the previous in vitro investigations by including a comparison of these devices in three different vessel diameters $(5,5.5$, and $6 \mathrm{~mm})$, with a statistical analysis to account for the Bonferroni correction in the multiple t-testing procedure.

\section{Methods}

\subsection{Experimental procedure}

Three distal protection devices were tested in a flow-loop system under simulated average systolic flow conditions using large polymer microspheres ranging in size from $297 \mu \mathrm{m}$ to $1000 \mu \mathrm{m}$ (mean $649 \mu \mathrm{m}$ ) and silicone tubes of different diameters (5.0, 5.5 and 6.0mm ID).

The inlet reservoir was filled with distilled water and a peristaltic roller pump (Cole Palmer Instruments, Vernon Hills, IL), pressure transducer (Omega Systems, Inc., Stamford, CT), and flow transducer (Transonic Systems, Inc., Ithaca, NY) were used to pump $180 \mathrm{~mL} / \mathrm{min}$ of the fluid at $80 \mathrm{mmHg}$. The distal protection device to be tested was then deployed, following the manufacturer's instructions, at a prescribed location within the test segment through a hemostatic valve connected to the flow-loop. A particle solution was prepared, consisting of dry, blue-dyed $\mathrm{P}(\mathrm{S} / 2 \% \mathrm{DVB})$ microspheres (Bangs Laboratory, Fishers, IN) with a density of $1.062 \mathrm{~g} / \mathrm{mL}$, distilled water, and Tween-20 (Fisher Scientific, Fairlawn, NJ). This solution was agitated with a magnetic stirrer before a $0.4 \mathrm{~mL}$ aliquot was drawn into a $10 \mathrm{~mL}$ syringe supplemented with the Tween-20, distilled water solution. Because of an appreciable standard deviation, the amount injected was verified by counting the particles in the $0.4 \mathrm{~mL}$ of solution under a BioScope with a surface indexed grid scale with a $0.1 \mathrm{~mm}$ side length, and the distal protection device effectiveness was determined based on the actual number of particles injected.

Once the particles were injected, the flow conditions were maintained for 5 minutes. To insure a complete injection, the piston of the syringe was released after 30 seconds with the flow-loop in operation, thereby refilling the syringe with the fluid from the system, and injected back into the system. This was repeated at another 30-second interval before keeping the piston of the syringe compressed through the remainder of the 5 minutes. At the end of this period the in-line filter A was removed and replaced by in-line filter B. The flow rate was reduced while the distal protection device was removed using the retrieval sheath 
through the hemostatic valve. The flow rate was restored and maintained for another minute to ensure all particles lost during the retrieval process were captured by in-line filter B. The latter was then removed and both in-line filters were placed in a drying oven at $37^{\circ} \mathrm{C}$. The particles from the device and dried inline filters were collected, counted, and recorded using randomized blinded counting. This procedure was repeated for $n=15$ trials for each device per test tube diameter. Table 1 describes the nominal size (expanded diameter) of the devices used and the manufacturer's recommended treatment range in terms of ICA internal diameter. The average pore size of the device membrane in these devices is $110 \mu \mathrm{m}$.

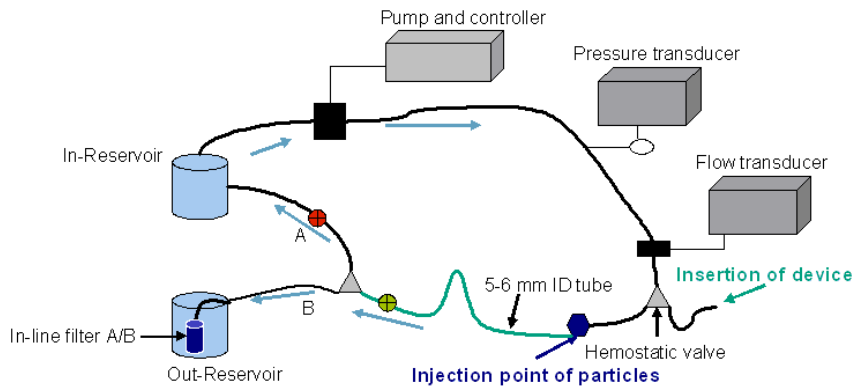

Figure 1: The in vitro flow-loop system used for this study $[17,18]$.

Table 1: Expanded device diameter and manufacturer's recommended treatment range $(\mathrm{mm})$.

\begin{tabular}{|c|c|c|c|}
\hline Test tube ID (mm) & Angioguard XP & RX Accunet & FilterWire EZ \\
\hline \multirow{2}{*}{5.0} & $\begin{array}{c}6.0 \\
(4.5<\mathrm{ID}<5.5)\end{array}$ & $\begin{array}{c}6.5 \\
(5.0<\mathrm{ID}<6.0)\end{array}$ & $\begin{array}{c}6.0 \\
(3.5<\mathrm{ID}<5.5)\end{array}$ \\
\hline \multirow{2}{*}{5.5} & $\begin{array}{c}6.0 \\
(4.5<\mathrm{ID}<5.5)\end{array}$ & $\begin{array}{c}6.5 \\
(5.0<\mathrm{ID}<6.0)\end{array}$ & $\begin{array}{c}6.0 \\
(3.5<\mathrm{ID}<5.5)\end{array}$ \\
\hline \multirow{2}{*}{6.0} & $\begin{array}{c}7.0 \\
(5.5<\mathrm{ID}<6.5)\end{array}$ & $(5.0<\mathrm{ID}<6.0)$ & $\mathrm{N} / \mathrm{A}^{1}$ \\
\hline
\end{tabular}

${ }^{1}$ FilterWire EZ is not manufactured for vessels with ID $>5.5 \mathrm{~mm}$.

\subsection{Statistical analysis}

Rather than considering all of the groups collectively and performing an analysis of variance, multiple t-test procedures were used for the statistical analysis. This was done to properly report the statistical findings of multiple experiments. Results reported from an analysis of variance may hide multiple testing procedures that are not conducted properly. On the other hand, it is known that a significant $F$ in analysis of variance is only an indication that not all the group means are equal. Once a significant value for the $F$-test is found in a standard analysis of variance, it is still unclear which ones are the groups presenting a smaller mean than the others, and further testing of more specific hypotheses needs to be conducted for isolating significant differences. Informal choices can 
be made, for example, by declaring that the group with the smaller average is the group corresponding to the best performing filter. But this type of practice is in fact equivalent to carrying out a multiple testing procedure, without acknowledging it explicitly.

A total of ten t-tests were considered to include all of the two-group comparisons matching for device type and size: three for evaluating the filter types, disregarding size, and seven more for comparing filters within the same size. An appropriate sample size can be determined by balancing the error associated with the measurement and the acceptable difference to be detected among the group of measurements [19]. That is to say that by following a standard calculation, the sample size $n$ required in each group for this test to have a power of $1-\beta$ for detecting a difference $\tau_{\mathrm{i}}$ in group $I$, is the value $n$ that satisfies $\mathrm{Eq}(1)$.

$$
n=I \sigma^{2} \frac{\phi^{2}}{\sum \tau_{i}^{2}}
$$

Let $\phi$ be the non centrality parameter of a non central $F$ distribution $\mathrm{f}_{\mathrm{d} 1, \mathrm{~d} 2}(\phi)$, found through the condition $\operatorname{Pr}\left(\mathrm{F}_{\mathrm{d} 1, \mathrm{~d} 2}(\phi)>\mathrm{F}_{\alpha, \mathrm{d} 1 \mathrm{~d} 2}\right)=1-\beta$. If $\alpha=0.05$ and $\beta=$ 0.368 , for a power of $0.632, \tau_{i}=0.9$ unit of weight difference in each group, and $\sigma=1.2$, the sample size of 15 patients per group is sufficient.

The significance level is defined to be the probability of making a type I error in the analysis, or the probability of concluding that the null hypothesis is false, when it is actually true. However, the significance level chosen has to be considered globally for the family of ten tests. Hence choosing $\alpha=0.05$ as the probability of a type I error in the analysis means fixing to 0.05 the probability of making one type I error in the family of ten tests. If $\alpha$ is the family-wise significance level, then the significance level of each test, in a set of $k$, is reduced to $\alpha^{\prime}=\alpha / k$. Therefore, the significance level of each of the ten t-tests is decreased to $\alpha^{\prime}=\alpha / 10=0.005$. This necessary reduction of the significance level of each test in a multiple-testing procedure is known as the Bonferroni correction. The Bonferroni correction illustrates one drawback of designing too many testing procedures in any given experiment. Conducting all the planned tests, while using the proper statistical approach, substantially $\mathrm{d}$ iminishes the significance level one is allowed to use in each test.

\section{Results and discussion}

\subsection{Weights of injected particles}

The values of means and standard deviations for the weight of the particles injected and collected in position A are presented for each group in Table 2. As evident from these results, the average weights of the injected microspheres are not stable across the groups. Thus, to verify that the differences in the average 
weights injected are due to chance and do not invalidate the experiment, a further F-test for equality of the means of injected weights was conducted, with a standard analysis of variance procedure. The null hypothesis tested was that the means of the injected weights among the three filters were equal, or H0: w1 = $\mathrm{w} 2=\mathrm{w} 3=\mathrm{w}$, where $\mathrm{w}$ is the unknown true value of the weight injected in each of the three groups. The result of this test shows that the differences in the weights injected are not statistically significant. The p-value, $\operatorname{Pr}(>F)$, is 0.02203 $>\alpha^{\prime}=0.005$. Thus we accept the null hypothesis that all means are equal, and this concern will not pose a challenge to our subsequent analysis.

Table 2: Mean weight of embolized particles that passed the distal protection devices in the test tube.*

\begin{tabular}{|c|c|c|c|}
\cline { 2 - 4 } \multicolumn{1}{c|}{} & $\mathbf{5 . 0 m m}$ & $\mathbf{5 . 5 m m}$ & $\mathbf{6 . 0 m m}$ \\
\cline { 2 - 4 } \multicolumn{1}{c|}{} & \multicolumn{3}{c|}{ Angioguard XP } \\
\hline $\mathbf{W}_{\text {in }}$ & $8.76 \pm 1.41$ & $9.93 \pm 3.27$ & $11.52 \pm 4.36$ \\
$\mathbf{W}_{\mathbf{A}}$ & $0.66 \pm 0.92$ & $1.08 \pm 1.48$ & $1.64 \pm 1.22$ \\
$\mathbf{R}_{\mathbf{A}}$ & $(8.08 \%)$ & $(11.83 \%)$ & $(16.73 \%)$ \\
\hline & \multicolumn{3}{c|}{ RX Accunet } \\
\hline $\mathbf{W}_{\text {in }}$ & $7.22 \pm 0.89$ & $9.58 \pm 2.34$ & $10.15 \pm 3.78$ \\
$\mathbf{W}_{\mathbf{A}}$ & $0.30 \pm 0.06$ & $0.02 \pm 0.08$ & $0.14 \pm 0.25$ \\
$\mathbf{R}_{\mathbf{A}}$ & $(0.42 \%)$ & $(0.16 \%)$ & $(2.13 \%)$ \\
\hline & \multicolumn{3}{c|}{ FilterWire EZ } \\
\hline $\mathbf{W}_{\text {in }}$ & $8.08 \pm 1.71$ & $8.38 \pm 1.93$ & -- \\
$\mathbf{W}_{\mathbf{A}}$ & $0.08 \pm 0.17$ & $0.05 \pm 0.12$ & -- \\
$\mathbf{R}_{\mathbf{A}}$ & $(1.07 \%)$ & $(1.01 \%)$ & -- \\
\hline
\end{tabular}

$* \mathrm{~W}_{\text {in }}=$ weight of injected particles (mg). $\mathrm{W}_{\mathrm{A}}=$ weight of emboli (mg). $\mathrm{R}_{\mathrm{A}}=\%$ of emboli. Data are expressed as mean \pm standard deviation and as a percentage of the originally injected particle weight in parenthesis. $\mathrm{W}_{\mathrm{A}}$ does not include emboli lost during retrieval of the device. The data were processed based on the microspheres manufacturer's certificate of analysis, which states that there are 6,579 particles/gram.

\subsection{Capture effectiveness}

The capture effectiveness $(\mathrm{R})$ of the devices is also shown in Table 2. The RX Accunet device had the single most overall filtration performance for these large PVA particles $(0.02 \mathrm{mg}$ and $0.16 \%$ for a tube diameter of $5.5 \mathrm{~mm})$. In a clinical setting, the target $\mathrm{R}_{\mathrm{A}}$ value would be $0 \%$ for all plaque particles of any size dislodged from the stenosis site. Therefore, none of the distal protection devices prevented embolization completely. These preliminary studies allow us to conclude that for embolic particles of mean diameter 6 times the pore diameter of the device membrane under constant luminal flow rate and pressure conditions in a compliant artery model of uniform cross-section, the devices tested did not perform satisfactorily as to completely reduce the risk of embolization and prevent stroke. Therefore, it can only be inferred that under in vivo conditions, where the emboli would include smaller particles, the carotid artery may be 
tortuous or with a nonuniform cross-section, and the luminal conditions may be adverse due to hypertension, the performance of these devices will be less than desirable in the prevention of stroke. Additionally, several intangible complications emerged from the continuous use of all the devices, namely (a) an abrupt change in stiffness and torqueability, (b) particles flowing around the device, (c) loss of particles during device retrieval, and (d) difficulties in retrieving the device. These complications may have been the result of limited operator training in the handling of the cerebral protection devices, as a student and not a medical professional was responsible for deploying and retrieving the filter from the flow-loop system. Further limitations of this experimental protocol include the lack of tortuosity or a bifurcation to the internal and external carotid arteries in the silicone tubing used, which may affect flow patterns and subsequent device performance. The constant, systolic flow rate supplied to the flow loop excludes the additional dynamics which may arise from the pulsatile blood flow found within the carotid artery. And while a broad distribution of particle sizes were used to emulate the dislodged arterial debris, the size of the microspheres captured in the filter devices or in the downstream in-line filters could not be quantified. Therefore, it is difficult to discern whether the various filter devices are able to capture smaller emboli, which may also induce neurological disorders.

\section{3 t-test and confidence interval}

As a more stable measure of the efficiency of the cerebral protection devices, the ratios between the weight of emboli collected in filter $\mathrm{A}$ and the weight of injected material were considered for this analysis. This fraction may also be represented as the percentage of emboli that the filter failed to capture and therefore the smaller the value, the better protection the filter offers for the procedure under the in vitro conditions.

Table 3 shows the results from the planned t-tests comparing the three filters disregarding vessel diameter, and then among the three tube diameters used in this study. Because of the Bonferroni correction, each test is done at a level $\alpha^{\prime}=\alpha / 10=0.005$. These results show that when testing the difference between the Angioguard XP and RX Accunet and between the Angioguard XP and FilterWire EZ, the p-values are respectively $6.76 \times 10^{-6}$ and $7.41 \times 10^{-6}$. Thus, we deduce that there is a significant overall difference in the ability to capture emboli among these devices when vessel diameter is not considered. However, testing for a difference between the RX Accunet and FilterWire EZ yielded a pvalue of $0.8187>\alpha^{\prime}$ and thus we can accept the null hypothesis that there is no significant difference on the performance of these dev ices.

A more detailed comparison among the devices and vessel diameter reveals that due to the low significance level needed for these tests, only the hypothesis of equal performance between the Angioguard XP and RX Accunet devices in the $6 \mathrm{~mm}$ diameter vessel can be rejected. All the other tests present p-values larger than $\alpha^{\prime}$ and the hypothesis of no difference in the performance of any of the devices for the 5 and $5.5 \mathrm{~mm}$ sizes can be accepted. This specific result is due in part to the fact that the experiment considered a large number of t-tests, and 
the correct significance level in each of them is quite small. Also note that the larger sample size (45 observations for each device) of the groups considered in Table 3 makes those results more decisive.

Table 3: t-test results by device and vessel inner diameter (ID) comparing the fraction of emboli the device failed to capture.

\begin{tabular}{|c|c|c|c|c|c|}
\hline \multicolumn{2}{|c|}{ p-value } & All tube IDs & $\begin{array}{c}5 \mathrm{~mm} \\
(n=15)\end{array}$ & $\begin{array}{l}5.5 \mathrm{~mm} \\
(n=15)\end{array}$ & $\begin{array}{c}6.0 \mathrm{~mm} \\
(n=15)\end{array}$ \\
\hline Angioguard $\mathrm{XP}^{2}$ & RX Accunet $^{2}$ & $6.76 \times 10^{-6}$ & 0.028 & 0.017 & 0.003 \\
\hline Angioguard $\mathrm{XP}^{2}$ & FilterWire $\mathrm{EZ}^{3}$ & $7.41 \times 10^{-6}$ & 0.051 & 0.019 & -- \\
\hline RX Accunet $^{2}$ & FilterWire $\mathrm{EZ}^{3}$ & 0.819 & 0.259 & 0.287 & -- \\
\hline
\end{tabular}

${ }^{2}$ measurements for all tube IDs totaled $45(n=45)$

${ }^{3}$ measurements for all tube IDs totaled $30(n=30)$

When the $99.5 \%$ confidence interval estimates for the differences in efficacy of the filters in the experiment were analyzed, both overall and by size, the results matched those from Table 3. The intervals were constructed at 100(1$\left.\alpha^{\prime}\right) \%$ confidence level, and were built using results from multiple t-testing. All of the t-tests in Table 3 that accepted the null hypothesis of no difference in ability to capture emboli among the devices and vessel sizes also included the point 0 in the $99.5 \%$ confidence interval, which is an indication that a zero difference in efficacy is a feasible value in the confidence interval. Some intervals are quite small around zero, which shows that no difference is well supported in the interval. Larger confidence intervals still support the possibility of a positive estimate of the difference. As mentioned before, the large number of t-tests planned in this experiment, the low value of $\alpha$, and the somewhat limited number of observations in these subgroups all contribute to the difficulty of identifying specific information when very detailed analyses are considered.

\section{Conclusions}

The RX Accunet and FilterWire EZ devices performed equally well, while Angioguard XP seems to have a lower cerebral protection effectiveness. The additional detailed analysis that divides the devices by size illustrates results that are less clear. In fact, the hypothesis of comparable performance between the Angioguard XP and RX Accunet and the Angioguard XP and FilterWire EZ for 5 and $5.5 \mathrm{~mm}$ cannot be rejected. Confidence intervals $(99.5 \%)$ illustrate this point as well, with small intervals between the RX Accunet and FilterWire EZ when compared by size, and larger intervals when Angioguard XP was compared with both the RX Accunet and FilterWire EZ, both globally and by size. None of the devices tested completely prevented embolization into the artery model. Overall, the RX Accunet device had the best filtration performance, failing to capture $0.16 \%$ of plaque particles when deployed in an artery model of $5.5 \mathrm{~mm}$ in diameter. Several complications related to device retrieval were detected in all devices on any given set of testing scenarios. 
Crossing profile, opening and closing mechanics, and pore size were among the key design variables requiring improvement for future generation devices.

\section{Acknowledgements}

This work was supported in part by the Pennsylvania Infrastructure Technology Alliance (PITA), a partnership of Carnegie Mellon, Lehigh University, and the Commonwealth of Pennsylvania's Department of Community and Economic Development (DCED), and a pre-doctoral fellowship by the Dowd-ICES Fellowship Program.

\section{References}

[1] Wholey, M.H., Wholey, M., Jarmolowski, C.R., and Eles, G.R., The growing role of carotid artery stenting, Endovascular Today, Sep. 2002.

[2] Diethrich, E.B., Indications for carotid stenting: a preview of the potential derived from early clinical experience, Journal of Endovascular Surgery, 3, pp. 132-139, 1996.

[3] Yadav, J.S., Roubin, G.S., King, P., Iyer, S., and Vitek, J., Angioplasty and stenting for restenosis after carotid endarterectomy, Stroke, 27, pp. 2075-2079, 1996.

[4] Roubin, G.S., New, G., Iyer, S.S., Vitek, J.J., Al-Mubarak, N., et al., Immediate and late clinical outcomes of carotid artery stenting in patients with symptomatic and asymptomatic carotid artery stenosis: a 5-year prospective analysis, Circulation, 103, pp. 532-537, 2001.

[5] Brooks, W.H., McClure, R.R., Jones, M.R., Coleman, T.C., and Breathitt, L., Carotid angioplasty and stenting versus carotid endarterectomy: randomized trial in a community hospital, Journal of the American College of Cardiology, 38, pp. 1589-1595, 2001.

[6] Deaton, D.H., Surgery or stenting?, Endovascular Today, Sep. 2002.

[7] Castriota, F., Cremonsi, A., Manetti, R., Liso, A., Oshola, K., et al., Impact of cerebral protection devices on early outcome of carotid stenting, Journal of Endovascular Therapy, 9, pp. 786-792, 2002.

[8] Reimers, B., Corvaja, N., Moshiri, S., Sacca, S., and Albiero, R., Cerebral protection with filter devices during carotid artery stenting, Circulation, 104, pp. 12-15, 2001.

[9] Wholey, M.H., Al-Mubarek, N., and Wholey, M.H., Updated review of the global carotid artery stent registry, Catheterization and Cardiovascular Interventions, 60, pp. 259-266, 2003.

[10] Ohki, T., Roubin, G.S., Veith, F.J., Iyer, S.S., and Brady, E., Efficacy of a filter device in the prevention of embolic events during carotid angioplasty and stenting: an ex vivo analysis, Journal of Vascular Surgery, 30, pp. 1034-1044, 1999.

[11] Bosiers, M., Peeters, P., Verbist, J., Schroe, H., Deloose, K., et al., Belgian experience with FilterWire EX in the Prevention of Embolic 
Events during Carotid Stenting, Journal of Endovascular Therapy, 10, pp. 695-701, 2003.

[12] Order, B.M., Glass, C., Liess, C., Heller, M., and Müller-Hülsbeck, S., Comparison of 4 cerebral protection filters for carotid angioplasty: an in vitro experiment focusing on carotid anatomy, Journal of Endovascular Therapy, 11, pp. 211-218, 2004.

[13] Müller-Hülsbeck, S., Grimm, J., Liess, C., Hedderich, J., Bergmeyer, M., and Heller, M., Comparison and modification of two cerebral protection devices used for carotid angioplasty: in vitro experiment, Radiology, 225, pp. 289-294, 2002.

[14] Müller-Hülsbeck, S., Jahnke, T., Liess, C., Glass, C., Paulsen, F., et al., In vitro comparison of four cerebral protection filters for preventing human plaque embolization during carotid interventions, Journal of Endovascular Therapy, 9, pp. 793-802, 2002.

[15] Müller-Hülsbeck, S., Jahnke, T., Liess, C., Glass, C., Grimm, J., and Heller, M., Comparison of various cerebral protection devices used for carotid artery stent placement: an in vitro experiment, Journal of Vascular and Interventional Radiology, 14, pp. 613-620, 2003.

[16] Müller-Hülsbeck, S., Hüsler, E., Schaffner, S., Jahnke, T., Glass, C., et al., An in vitro analysis of a carotid artery stent with a protective porous membrane, Journal of Vascular and Interventional Radiology, 15, pp. 1295-1305, 2004.

[17] Scotti, C.M., Azemi, E., Gray, H.D., Miner, E.P., Wholey, M.H., and Finol, E.A., Efficiency of cerebral protection devices for carotid angioplasty and stenting, 2004 Biomedical Engineering Society Annual Fall Meeting, Philadelphia, PA, October 13-16, 2004.

[18] Finol, E.A., Scotti, C.M., Verdinelli, I., and Wholey, M.H., On the effectiveness of distal protection devices in carotid artery stenting, Proceedings of ASME 2005 Summer Bioengineering Conference, Vail, CO, June 22-26, 2005, in press.

[19] Howell, D.C., Statistical Methods for Psychology, PWS-KENT Publishing Company, Boston, 1992. 